\title{
3 Research Square

\section{Fermi Blockade of the Electron-phonon Interaction: Why Strong Coupling Effects May Not Be Seen in Optimally Doped High Temperature Superconductors}

\author{
Andrey Mishchenko ( $\square$ mishchenko@riken.jp) \\ RIKEN Center for Emergent Matter Science (CEMS) \\ Naoto Nagaosa \\ RIKEN Center for Emergent Matter Science \\ Nikolay Prokof'ev \\ University of Massachusetts
}

\section{Article}

Keywords: Electron-phonon Interaction, High Temperature

Posted Date: September 16th, 2020

DOI: https://doi.org/10.21203/rs.3.rs-73364/v1

License: (c) (i) This work is licensed under a Creative Commons Attribution 4.0 International License.

Read Full License 


\title{
1 Fermi blockade of the electron-phonon interaction: why strong coupling effects may not be seen in optimally doped high temperature superconductors.
}

\author{
Andrey S. Mishchenko*1,2, Naoto Nagaosa ${ }^{1,3}$, and Nikolay Prokof' ${ }^{4,2}$ \\ ${ }^{1}$ RIKEN Center for Emergent Matter Science (CEMS), Wako, Saitama 351-0198, \\ Japan \\ ${ }^{2} \mathrm{NRC}$ "Kurchatov Institute", 123182 Moscow, Russia \\ ${ }^{3}$ Department of Applied Physics, The University of Tokyo 7-3-1 Hongo, \\ Bunkyo-ku, Tokyo 113-8656, Japan \\ ${ }^{4}$ Department of Physics, University of Massachusetts, Amherst, MA 01003, USA
}

We study how manifestations of strong electron-phonon interaction (EPI) depend on the carrier concentration by solving the two-dimensional Holstein model for the spin-polarized fermions using an approximation free bold-line diagrammatic Monte Carlo (BDMC) method. We show that the strong EPI, obviously present at very small Fermion concentration, is masked by the Fermi blockade effects and Migdal's theorem to the extent that it manifests itself as moderate one at large carriers densities. Suppression of strong EPI fingerprints is in agreement with experimental observations in doped high temperature superconductors.

Discussions on the role of the EPI in the physics of cuprate compounds with high superconducting transition temperatures (high $T_{c}$ ) have been going for decades $[1,2,3,4,5,6]$ without resulting in a consensus opinion. While the role of EPI in superconductivity is still under debate, its strong manifestations were clearly observed in numerous other phenomena in high $T_{c}$ materials $[5,6,7,8,9,10,11$, $12,13,14]$. The apparent puzzle is that strong EPI effects seen in spectroscopic data of undoped and weakly doped compounds become much less pronounced with hole doping $[15,16,17,18]$. Hence, having a clear picture of how the EPI effects change with the carrier concentration is of seminal importance for understanding the nature of unconventional superconductors where rigorous studies are hindered by the complexity of many-body fermion problem. Accurate results on the EPI in many-fermion systems may provide the way to reconcile the observed fingerprints of the strong EPI in the underdoped regime with successful descriptions of the strongly doped high $T_{c}$ materials by models based on direct electron-electron interactions alone.

More generally, it is a long standing fundamental problem to reveal how the Migdal's theorem [19, 20] emerges at the large fermion concentration and eliminates the need for vertex corrections even for strong EPI, provided the Fermi-liquid state remains stable. The crossover between the two regimes is expected to take place at $\omega_{\mathrm{ph}} \sim \varepsilon_{F}$, where $\omega_{\mathrm{ph}}$ is the phonon frequency and $\varepsilon_{F}$ is the Fermi energy, and it can be addressed by the approximation diagrammatic Monte Carlo methods $[21,22,8,23]$. To this end, we consider a spin polarized (SP) two-dimensional (2D) lattice system in order to avoid system instabilities that would be triggered by the strong EPI in continuous and spin-balanced systems, such as structural transitions 45 or a singlet on-site bipolaron formation at $\lambda \approx 0.5$ (in 2D) [24] with the concomitant 
The 2D Holstein model on a square lattice reads:

$$
H=-t \sum_{<i, j>} c_{i}^{\dagger} c_{j}+\omega_{\mathrm{ph}} \sum_{i} b_{i}^{\dagger} b_{i}+g \sum_{i} c_{i}^{\dagger} c_{i}\left(b_{i}^{\dagger}+b_{i}\right),
$$

superconducting state. An essential feature of the SP model resembling that of the $t-J$ model near half-filling $[25,26]$ (which is prototypical for description of high $T_{c}$ superconductors) is that in both cases one can only create one hole per site.

In this work we employ the BDMC technique developed for many-body systems with EPI in Ref. [23]. For the same system parameters the determinant Monte Carlo [27, 28] method would suffer from a severe sign problem. The dynamical mean-filed method (DMFT) [29, 30], would be inadequate because the EPI self-energy is strongly momentum dependent at low carrier concentration [23], in violation of the key DMFT assumption. The BDMC technique is based on the expansion of irreducible free-energy Feynman diagrams in terms of exact electron, $G$, and bare, $D^{(0)}$, phonon propagators [?] and is free from the above limitations. Here, we neglect renormalization of phonon propagators $D^{(0)}$ because the rigorous treatment of phonon formation requires accurate ab-initio treatment $[31,32]$ and has no significant influence on our results, partially due to small concentration of carriers in the most interesting regime. In more detail, see Ref. [23], the electron self-energy $\Sigma^{(m)}$ is expanded into the series of irreducible skeleton graphs up to the largest order $m$ defined by the number of $D^{(0)}$ propagators, with selfconsistency implemented by a feedback loop involving the solution of the algebraic Dyson equation, $\left[G\left(\mathbf{k}, \omega_{\ell}\right)\right]^{-\mathbf{1}}=\left[\mathbf{G}^{(\mathbf{0})}\left(\mathbf{k}, \omega_{\ell}\right)\right]^{-\mathbf{1}}-\boldsymbol{\Sigma}^{(\mathbf{m})}\left(\mathbf{k}, \omega_{\ell}\right)$, in momentum, $\mathbf{k}$, and Matsubara frequency, $\omega_{\ell}=2 \pi T(\ell+1 / 2)$, representation ( $\ell$ is an integer).

where $c_{i}^{\dagger} / b_{i}^{\dagger}$ are standard notations for electron/phonon creation operators, $t$ is the nearest neighbor hopping amplitude, $\omega_{\mathrm{ph}}=0.5 t$ is the energy of the local optical mode, and $g$ is the EPI coupling. The electron gas is spin-polarized and, hence, any site can be occupied by no more than one electron. It is standard to characterize the strength of the EPI by a dimensionless coupling constant $\lambda=g^{2} /\left(4 \omega_{\mathrm{ph}} t\right)$. The lattice constant $a$, amplitude $t$, and Planck's constant $\hbar$ are used to set units of length, energy, and time, respectively. In this study we chose $\lambda=1.07$ beyond the crossover from weak- to strong-coupling regimes for single polarons and the threshold for the singlet bipolaron formation. For convenient systematic errorfree handling of the data in momentum space we perform simulations for finite systems with $16 \times 16$ sites, large enough to reproduce the infinite system results with high accuracy (see Supplemental Material [33]). The temperature is set to $T=t / 20$, which is an order of magnitude smaller than all energy scales of the model parameters. In the zero-density limit an alternative exact (numerically) diagrammatic Monte Carlo (DMC) approach for single polarons [21, 22] provides reference values for the ground state energy, $E_{1}=-4.891$, and the quasiparticle $(\mathrm{QP})$ residue, $Z_{1}=0.238$. 


\section{Results}

Our main results are presented in Figs. 1, 2, and 3. Figure 1 shows the dependence of the QP residue on the adiabaticity ratio $\gamma=\varepsilon_{F} / \omega_{\text {ph }}$. One can see in Fig. 1 that at large $\gamma \geq 3$ the Migdal's theorem ensures that vertex corrections are small and the lowest-order $m=1$ skeleton diagram for self-energy (also known, depending on the context, as the non-crossing, the self-consistent Born, and the Eliashberg approximations) well describes the EPI renormalization even at strong coupling. In contrast, for smaller values of $\gamma$ one has to account for high-order vertex corrections; up to order $m=7$ at $\gamma=1$ and all the way to $m>20$ for $\gamma \rightarrow 0$ with extrapolation to the infinite diagram-order limit. An immediate conclusion is that EPI strongly suppresses the QP residue to values smaller that 0.5 (indicative of strong coupling) only at a rather small filling factor when $\gamma<1$.

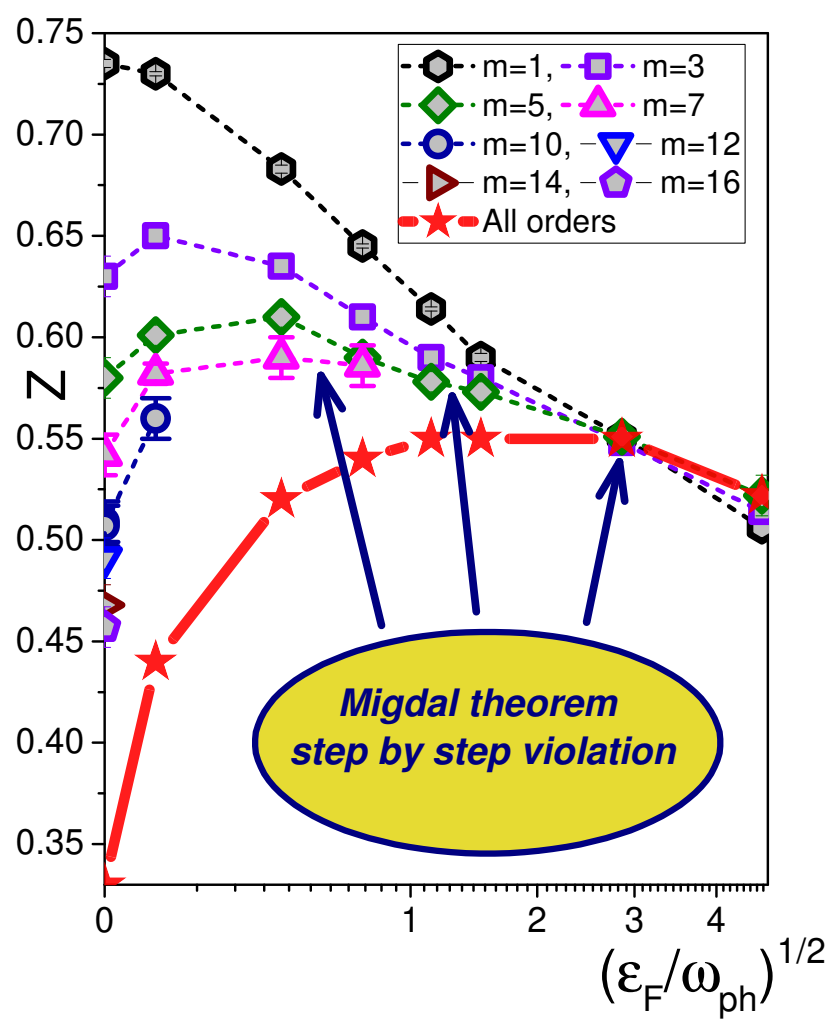

Figure 1. Quasi-particle residue at the Fermi (FS) as a function of ratio between the Fermi energy and phonon frequency without $(m=1)$ and with vertex corrections $(m>1)$. Symbols and dashed lines represent data obtained by skeleton expansions truncated at some finite order $m$. The solid red line with stars is obtained by extrapolation to the infinite diagram-order limit $m \rightarrow \infty$. The errorbars, if not visible, are smaller than the symbol sizes. 

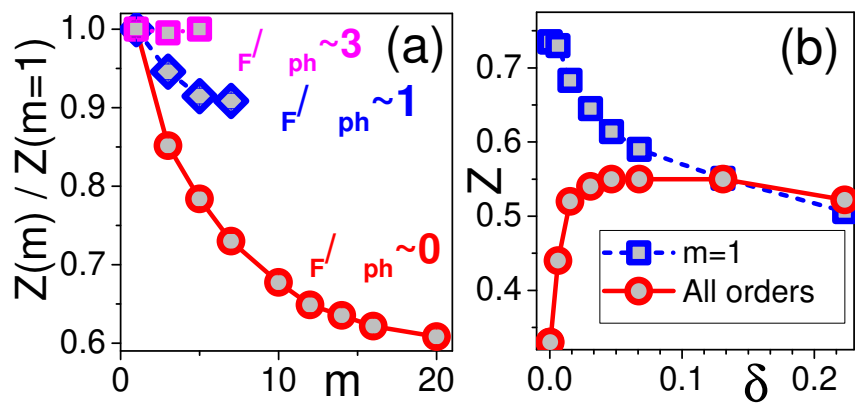

Figure 2. (a) Ratio between the quasi-particle residue deduced from diagrams up to order $m$ and $m=1$ (neglecting vertex corrections). Circles, diamonds, and squares stand for $\gamma \rightarrow 0$ $\left(\delta=3.8 \times 10^{-4}\right), \gamma=0.71(\delta=0.0308)$, and $\gamma=2.86(\delta=0.131)$, respectively. (b) Quasi-particle residue at the Fermi (FS) as a function of carrier concentration $\delta$ (circles, infinite diagram-order limit) in comparison with the $m=1$ result (squares), see also Fig. 1.

In Fig. 2(a) we further quantify the role of vertex corrections at low and high carrier density (or occupation number per site), $\delta$, in both adiabatic and antiadiabatic regimes. Vertex corrections become important at $\gamma<3$, and at low values of $\gamma$ and $\delta$ it is not sufficient to take into account just $m=2$, or even $m=3$ contributions; in this parameter regime the convergence is reached only for $m \geq 16$ in the skeleton expansion, see Fig. 2(a)). Figure 2(b) is complementary to Fig. 1 by presenting the data as a function of the carrier concentration $\delta$ instead of $\gamma$. Signatures of strong EPI are observed at $\delta<0.1$ that roughly corresponds to $\gamma \approx 1$. The key conclusion that clear manifestations of strong EPI are limited to small doping is consistent with experimental findings for high $T_{c}$ superconductors $[15,16,17,18]$.

One evidence for Fermi blockade of the EPI with doping comes from angle resolved photoemission experiments [15]. It was shown that the kink angle, related to the ratio, $v_{\text {high }} / v_{\text {low }}$, between the phase velocities of the dispersion relation above and below the Debye frequency, decreases with doping. Our simulations reveal a similar trend, see Fig. 3. The QP dispersion relation $\omega(\mathbf{k})$ was obtained from the energy of the lowest peak in the Lehmann spectral function, see Fig. 4, extracted from the imaginary time Matsubara Green function $G(\tau)$ by the stochastic optimization with consistent constraints method of analytic continuation [22, 34].

Calculations of the frequency dependent optical conductivity [16] and angle resolved photoemission spectra [18] in the low-concentration limit (one hole) of the $t-J$-Holstein model revealed that the experimental dependence of both quantities on $\delta$ can be reproduced theoretically if one introduces effective EPI coupling constant $\lambda^{e}(\delta)$ that decreases with doping. It can be deduced from the photoemission spectra using scaling relation [18]

$$
\lambda^{e}=\sqrt{\frac{v_{\text {high }}-v_{\text {low }}}{20 v_{\text {low }}}},
$$




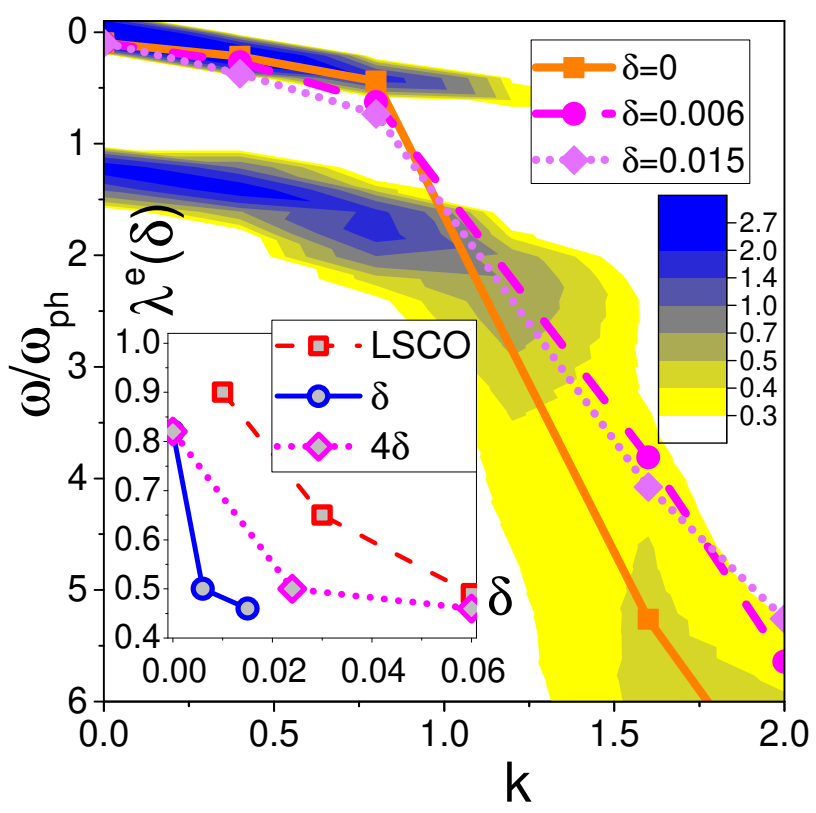

Figure 3. Contour plot of the spectral function intensity at $\delta=$ $3.8 \times 10^{-4}$ with blue/yellow color used for the large/small intensity. Symbols connected with lines mark locations of the spectral density maxima, see also Fig. 4 , for: $\delta=3.8 \times 10^{-4}$ (squares connected by the solid line), $\delta \approx 0.006$ (circles connected by the dashed line), and $\delta \approx 0.015$ (diamonds connected by the dotted line). In the inset we present the effective coupling constant $\lambda^{e}$ deduced from the scaling relation (2) using experimental data for LSCO [18] (squares connected by a dashed line) and locations of theoretical spectral density maxima in Fig. 4 (circles connected by a solid line). We also re-plot the same theoretical data by using $4 \delta$ for the horizontal axis (diamonds connected by a dotted line). Spectral densities were computed for self-energies evaluated up to order $m=16$ $\left(\delta=3.8 \times 10^{-4}\right), m=7(\delta=0.006)$, and $m=5(\delta=0.015)$. These expansion orders are enough to have converged results for the corresponding carrier density (see Supplemental Material [33], Table I).

derived from nonperturbative calculations for the $t-J$-Holstein model, where $v_{\text {low }}$ $\left(v_{\text {high }}\right)$ is the velocity above (below) the kink energy $\omega_{\text {ph }}$. Note, the doubling of the spectral peak around the kink energy $\omega_{\text {ph }}$ is a general feature of theoretical calculations $[7,35,36,18]$. These two peaks merge into a customary experimental picture of a single-peak kink at $\omega=\omega_{\text {ph }}$ when the theoretical spectra are broadened by experimental resolution or additional damping processes [35, 18]. We compare $\lambda^{e}(\delta)$ deduced from experimental data of Ref. [18] with our theoretical analysis in 


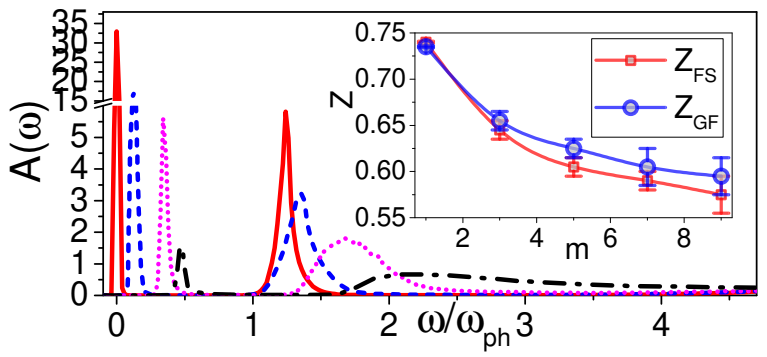

Figure 4. Spectral functions $A(\omega)$ at different momenta for $\delta=$ $3.8 \times 10^{-4}$ from $m=16$ simulations: $q=(0,0)$ (red solid line), $q=$ $(\pi / 8,0)$ (blue dashed line), $q=(2 \pi / 8,0)$ (magenta dotted line), and $q=(3 \pi / 8,0)$ (black dash-dotted line). Energy zero was set at the value of the QP dispersion relation at $q=0$. Inset: Orderby-order comparison between the two alternative procedures for computing the quasi-particle residue at $q=0$ : (i) using standard Fermi liquid relations at the Fermi surface, $Z_{F S}$, and (ii) from the lowest-frequency peak in the spectral function, $Z_{G F}$.

the inset of Fig. 3, dashed versus solid line. To have a meaningful quantitative comparison we also need to account for the difference between the non-degenerate spectrum of the spin-polarized Holstein model and fourfold degenerate ground state minimum of the experimental system. To this end we re-plot theoretical data using $4 \delta$ for the carrier concentration (dotted line). We observe semi-quantitative agreement between the theory and experiment despite a number of significant differences between the two cases at the microscopic level.

\section{Discussion}

The violation of Migdal's theorem for $T=0$ is apparent in Fig. 1 for all filling factors except the two largest ones. At the lowest carrier concentrations the condition $\varepsilon_{F} \gg T$ does not hold any more, but this fact is barely relevant for the discussion because the theorem is severely violated well before that, at $\varepsilon_{F} \sim \omega_{\mathrm{ph}} \gg T$. Thus, our finite temperature results are still valid for interpretation of the EPI suppression in high $T_{c}$ materials, which is observed from low to room temperatures $[15,16,17,18]$.

We computed approximation-free results for the concentration dependence of the quasiparticle residue $Z$ and kink angle caused by the strong electron-phonon interaction in the spin-polarized two-dimensional Holstein model on the square lattice. We demonstrated that clear signatures of strong electron-phonon coupling at small carrier concentration are quickly suppressed for Fermi energies exceeding the phonon frequency. Our results provide detailed account for importance of high-order vertex corrections across the adiabatic crossover and demonstrate that Fermi blockade of the electron-phonon interaction and irrelevance of vertex corrections both proceed in agreement with the Migdal's theorem. This picture explains experimental results reporting radical weakening of the electron-phonon coupling effects in lightly doped high temperature superconductors. 


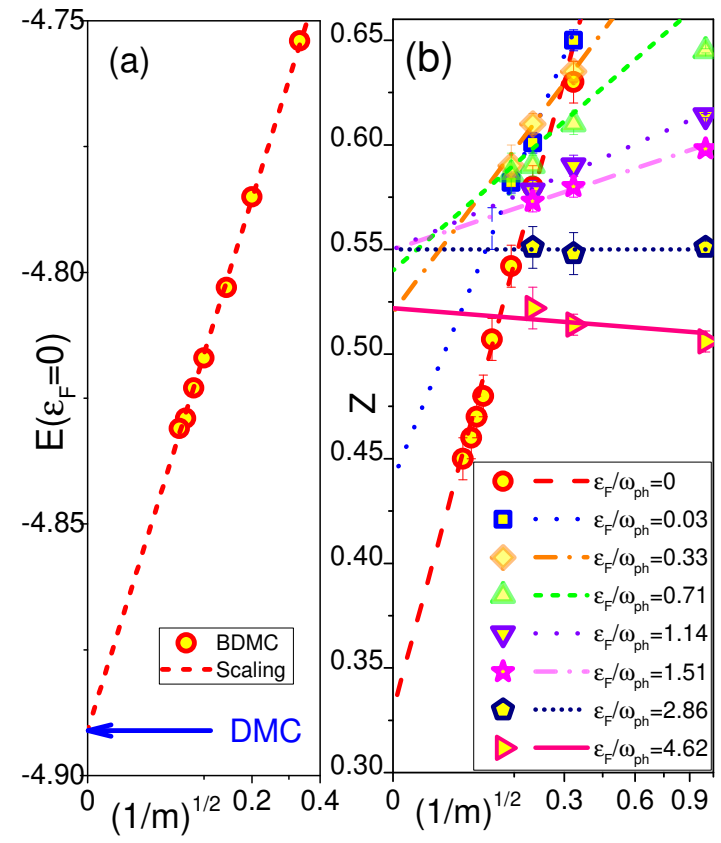

FiguRe 5. Finite expansion-order corrections to the polaron energy (a) and QP residue (b) revealing linear scaling with $m^{-1 / 2}$. (a) BDMC data (circles) and the scaling law $a+b / \sqrt{m}$ (dashed line) for the ground state energy at $\delta=3.8 \times 10^{-4}$. The DMC result at $\delta=0$ is shown by the blue arrow. (b) BDMC data (symbols) and the scaling laws $a+b / \sqrt{m}$ (lines) for the quasi-particle residue.

\section{Methods}

All data for the QP residues at the FS, also denoted as $Z_{F S}$, were deduced from the Fermi-liquid relation, $Z_{F S}=[1+d]^{-1}$, with $d=\partial \operatorname{Re}\left[\Sigma\left(k_{F}, \omega\right)\right] /\left.\partial \omega\right|_{\omega=0}$. In the low-temperature limit, the self-energy derivative at zero frequency is accurately obtained from the ratio $-\operatorname{Im}\left[\Sigma\left(k_{F}, \ell\right)\right] / \omega_{\ell}$ at the lowest Matsubara frequencies. As expected, this procedure works perfectly at large carrier concentration. However, in the zero density limit the Fermi surface shrinks to a point at zero momentum, and the entire protocol becomes questionable. Spectral density offers an alternative way of computing the QP residue from the integrated weight of the lowest frequency peak (we denote it as $Z_{G F}$ ), see Fig. 4. Somewhat surprisingly, we find that even in the zero-density limit both procedures produce consistent results at any expansion order $m$, see inset in Fig. 4. At small, but finite concentration $\delta=0.01526$ (or $\gamma=0.334)$, with Fermi-momentum $k_{F} \approx \pi / 8$ the agreement is even more precise: at order $m=5$ we find that $Z_{F S}=0.605$ and $Z_{G F}\left(k_{F}=\pi / 8\right)=0.611$.

As already mentioned in connection with Figs. 1 and Fig. 2(a), at small doping the skeleton expansion needs to go beyond $m=16$ in order to obtain correct results for the QP residue. However, both $Z$ and the polaron energy $E$ at the FS accurately follow an empirical scaling relation, $a+b / \sqrt{m}$, at any carrier concentration $\delta$, see 
Fig. 5. This allows us to perform an extrapolation to the infinite-order limit to eliminate the remaining systematic error as shown in Figs. 1-2. The extrapolation procedure is validated by an excellent agreement between the BDMC result for the ground state energy of single-polarons, $E(m \rightarrow \infty)=-4.89$ and the DMC benchmark $E_{1}=-4.891$. The single polaron zero temperature residue $Z_{1}=0.238$ is renormalized to $Z_{1}(\beta=20) \approx 0.31$ due to finite temperature projection of the low energy self-trapping states [37, 38] (see Supplemental Material [33]) which is also consistent with extrapolated value $Z(m \rightarrow \infty) \approx 0.33$.

\section{DATA AVAILABILITY}

The data presented are available from the corresponding authors upon reasonable request

\section{REFERENCES}

[1] Anderson, P. W. The Theory of Superconductivity in the High-T $T_{c}$ Cuprate Superconductors. (University Press, Princeton, 1997)

[2] Alexandrov, A. S. Bipolaron anisotropic flat bands, Hall mobility edge, and metalsemiconductor duality of overdoped high- $T_{c}$ oxides. Phys. Rev. B. 53, 2863-2869 (1996).

[3] Anderson, P. W. Is There Glue in Cuprate Superconductors? Science. 316, 1705-1707 (2007).

[4] Alexandrov, A. S. Bose-Einstein condensation of strongly correlated electrons and phonons in cuprate superconductors. Journal of Physics: Condensed Matter. 19, 125216 (2007).

[5] Gunnarsson, O \& Rösch, O. Interplay between electron-phonon and Coulomb interactions in cuprates. Journal of Physics: Condensed Matter. 20, 043201 (2008).

[6] Mishchenko, A. S. Electron-phonon coupling in underdoped high-temperature superconductors. Physics-Uspekhi. 52, 1193-1212 (2009).

[7] Rösch, O.\& Gunnarsson, O. Electron-Phonon Interaction in the $t-J$ Model. Phys. Rev. Lett. 92, 146403 (2004).

[8] Mishchenko, A. S. \& Nagaosa, N. Electron-Phonon Coupling and a Polaron in the $t-J$ Model: From the Weak to the Strong Coupling Regime. Phys. Rev. Lett. 93, 036402 (2004).

[9] Rösch, O., et al. Polaronic Behavior of Undoped High- $T_{c}$ Cuprate Superconductors from Angle-Resolved Photoemission Spectra. Phys. Rev. Lett. 95, 227002 (2005).

[10] Cataudella, V., De Filippis, G., Mishchenko, A. S. \& Nagaosa, N. Temperature Dependence of the Angle Resolved Photoemission Spectra in the Undoped Cuprates: Self-Consistent Approach to the $t-J$ Holstein Model. Phys. Rev. Lett. 99, 226402 (2007).

[11] De Filippis, G., Cataudella, V., Mishchenko, A. S. \& Nagaosa, N. Nonlocal Composite SpinLattice Polarons in High Temperature Superconductors. Phys. Rev. Lett. 99, 146405 (2007).

[12] De Filippis, G. et al. Quantum Dynamics of the Hubbard-Holstein Model in Equilibrium and Nonequilibrium: Application to Pump-Probe Phenomena. Phys. Rev. Lett. 109, 176402 (2012).

[13] Novelli, F. et al. Witnessing the formation and relaxation of dressed quasi-particles in a strongly correlated electron system. Nature Communications 5, 5112 (2014).

[14] Farina, D. et al. Electron-phonon coupling in the undoped cuprate $\mathrm{YBa}_{2} \mathrm{Cu}_{3} \mathrm{O}_{6}$ estimated from Raman and optical conductivity spectra. Phys. Rev. B. 98, 121104 (2018).

[15] Lanzara, A. et al. Evidence for ubiquitous strong electron-phonon coupling in hightemperature superconductors. Nature 412, 510-514 (2001).

[16] Mishchenko, A. S. et al. Charge Dynamics of Doped Holes in High $T_{c}$ Cuprate Superconductors: A Clue from Optical Conductivity. Phys. Rev. Lett. 100, 166401 (2008).

[17] Carbone, F, Yang, D.-S., Giannini, E., \& Zewail, A. H. Direct role of structural dynamics in electron-lattice coupling of superconducting cuprates. PNAS 105, 20161-20166 (2008).

[18] Mishchenko, A. S., Nagaosa, M. Shen, N., K.. Shen, Z.-X., Zhou, X. J., \& Devereaux, T. P. Polaronic metal in lightly doped high- $\mathrm{T}_{c}$ cuprates. EPL 95, 57007 (2011).

[19] Migdal, A. B. Interaction between electrons and lattice vibrations in a normal metal. J. Exptl. Theoret. Phys. 34 1438-1446 (1958) [Sov. Phys. JETP 7, 996-1001 (1958)].

[20] Husanu, M.-A. et al. Electron-polaron dichotomy of charge carriers in perovskite oxides. Communications Physics 3, 62 (2020). 
[21] Prokof'ev, N. V. \& Svistunov, B. V. Polaron Problem by Diagrammatic Quantum Monte Carlo. Phys. Rev. Lett. 81, 2514-2517 (1998).

[22] Mishchenko, A. S., Prokof'ev, N. V., Sakamoto, A. \& Svistunov, B. V. Diagrammatic quantum Monte Carlo study of the Fröhlich polaron. Phys. Rev. B 62, 6317-6336 (2000).

[23] Mishchenko, A. S., Nagaosa, N. \& Prokof'ev, N. Diagrammatic Monte Carlo Method for Many-Polaron Problems. Phys. Rev. Lett. 113, 166402 (2014).

[24] Macridin, A., Sawatzky, G. A. \& Jarrell, M. Two-dimensional Hubbard-Holstein bipolaron. Phys. Rev. B 69, 245111 (2004).

[25] Kane, C. L., Lee, P. A. \& Read, N. Motion of a single hole in a quantum antiferromagnet. Phys. Rev. B 39, 6880-6897 (1989).

[26] Dagotto, E. Correlated electrons in high-temperature superconductors. Rev. Mod. Phys. 66, 763-840 (1994).

[27] Blankenbecler, R., Scalapino, D. J. \& Sugar, R. L. Monte Carlo calculations of coupled boson-fermion systems. I. Phys. Rev. D 24, 2278-2286 (1981).

[28] Noack, R. M. \& Scalapino, D. J. Green's-function self-energies in the two-dimensional Holstein model. Phys. Rev. B 47, 305-308 (1993).

[29] Georges, A., Kotliar, G., Krauth, W. \& Rozenberg, M. J. Dynamical mean-field theory of strongly correlated fermion systems and the limit of infinite dimensions. Rev. Mod. Phys. 86, 13-125 (1996).

[30] Bauer, J., Han, J. E. \& Gunnarsson, O. Quantitative reliability study of the MigdalEliashberg theory for strong electron-phonon coupling in superconductors. Phys. Rev. B 84, 184531 (2011).

[31] Brovnman, E. G. \& Kagan, Yu. The Phonon Spectrum of Metals. J. Exptl. Theoret. Phys. 52 557-574 (1967) [Sov. Phys. JETP 25, 365-376 (1967)]

[32] Tupitsyn, I. S., Mishchenko, A. S., Nagaosa, N. \& Prokof'ev, N. Coulomb and electron-phonon interactions in metals. Phys. Rev. B 94, 155145 (2016).

[33] See supplemental material.

[34] Goulko, O., Mishchenko, A. S., Pollet, L., Prokof'ev, N. \& Svistunov, B. Numerical analytic continuation: Answers to well-posed questions. Phys. Rev. B 95, 014102 (2017).

[35] Devereaux, T. P., Cuk, T., Shen, Z.-X. \& Nagaosa, N. Anisotropic Electron-Phonon Interaction in the Cuprates. Phys. Rev. Lett. 93, 117004 (2004).

[36] Veenstra, C. N., Goodvin, G. L., Berciu, M. \& Damascelli, A. Elusive electron-phonon coupling in quantitative analyses of the spectral function. Phys. Rev. B 82, 012504 (2010).

[37] Bonča, J. and Trugman, S. A. \& Batistić I. Holstein polaron. Phys. Rev. B 60, 1633-1642 (1999).

[38] Mishchenko, A. S., Nagaosa, N., Prokof'ev, N. V., Sakamoto, A. \& Svistunov, B. V. Selftrapping of polarons in the Rashba-Pekar model. Phys. Rev. B 66, 020301 (2002).

\section{AcKnowledgements}

N.N. and A.S.M acknowledge support by JST CREST Grant Number JPMJCR1874, Japan, and N.P. acknowledges support of National Science Foundation under the grant DMR-1720465 and the Simons Collaboration on the Many Electron Problem

\section{Author contributions}

A.S.M. and N.P. developed a Diagrammatic Monte Carlo code. A.S.M. and N.N. conceived the idea of calculations. A.S.M. performed calculations and wrote the manuscript. All authors discussed the results, interpretations, and scientific concepts.

\section{Competing interests}

The authors declare no competing interests. 
275

276

277

\section{AdDitional information}

Supplementary information is available for this paper at https://?????

Correspondence and requests for materials should be addressed to A.S.M. 
Figures

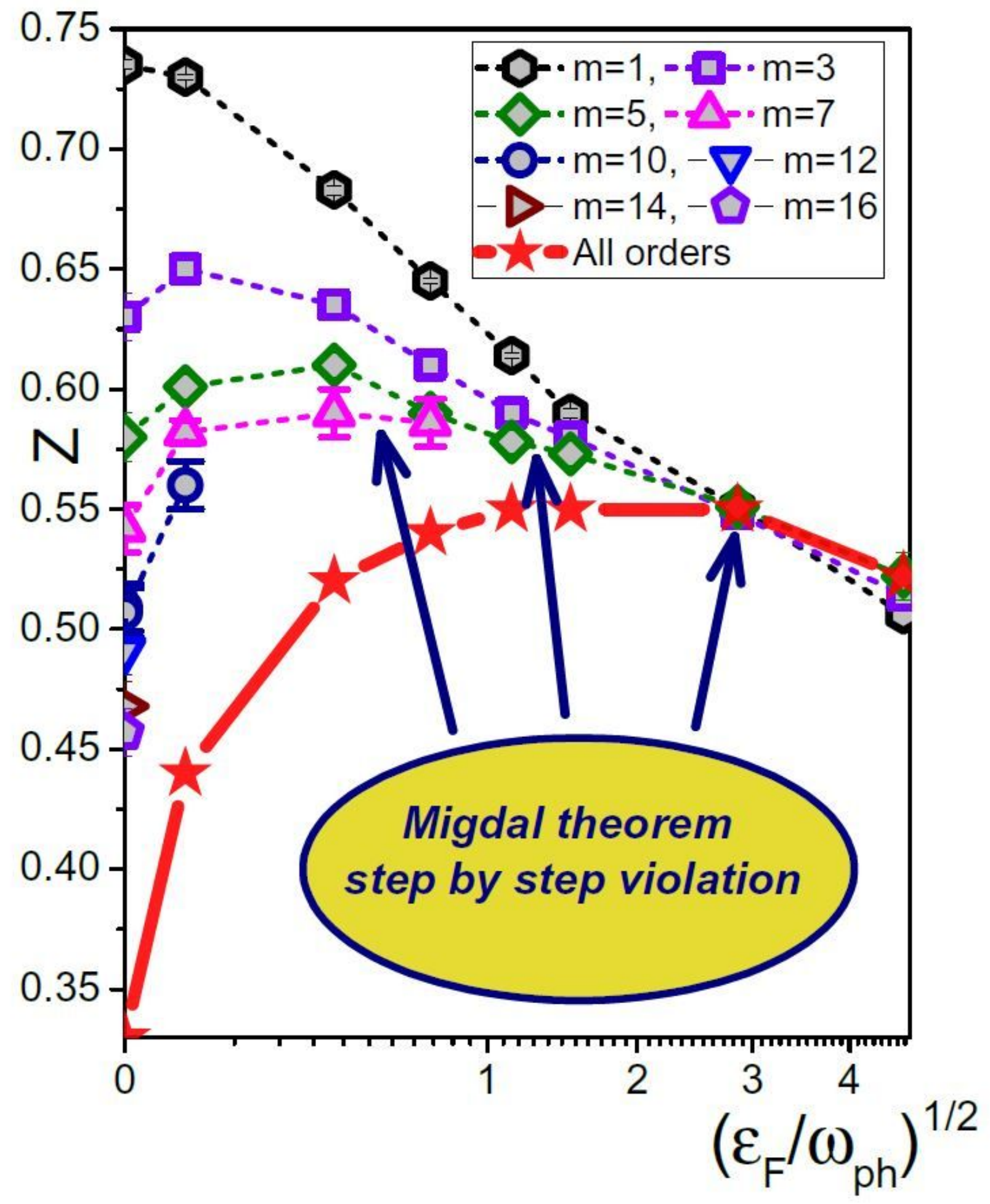

Figure 1

Quasi-particle residue at the Fermi (FS) as a function of ratio between the Fermi energy and phonon frequency without $(m=1)$ and with vertex corrections $(m>1)$. Symbols and dashed lines represent data obtained by skeleton expansions truncated at some finite order $\mathrm{m}$. The solid red line with stars is 

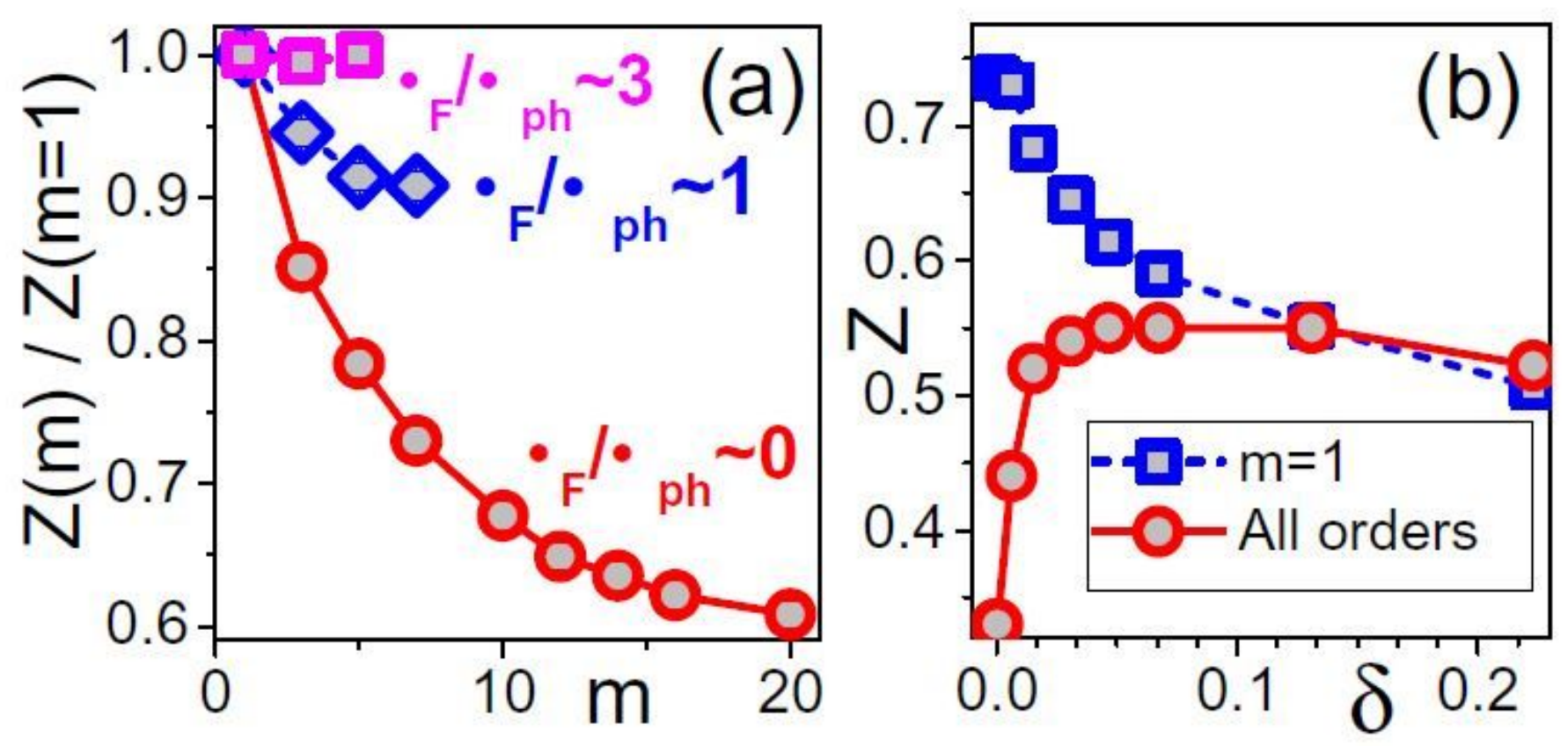

Figure 2

(a) Ratio between the quasi-particle residue deduced from diagrams up to order $\mathrm{m}$ and $\mathrm{m}=1$ (neglecting

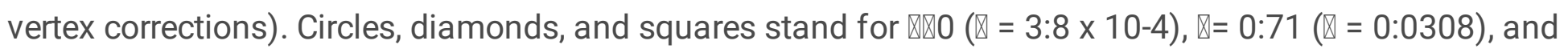
$\nabla=2: 86(\nabla=0: 131)$, respectively. (b) Quasi-particle residue at the Fermi (FS) as a function of carrier concentration $\otimes$ (circles, infinite diagram-order limit) in comparison with the $m=1$ result (squares), see also Fig. 1. 


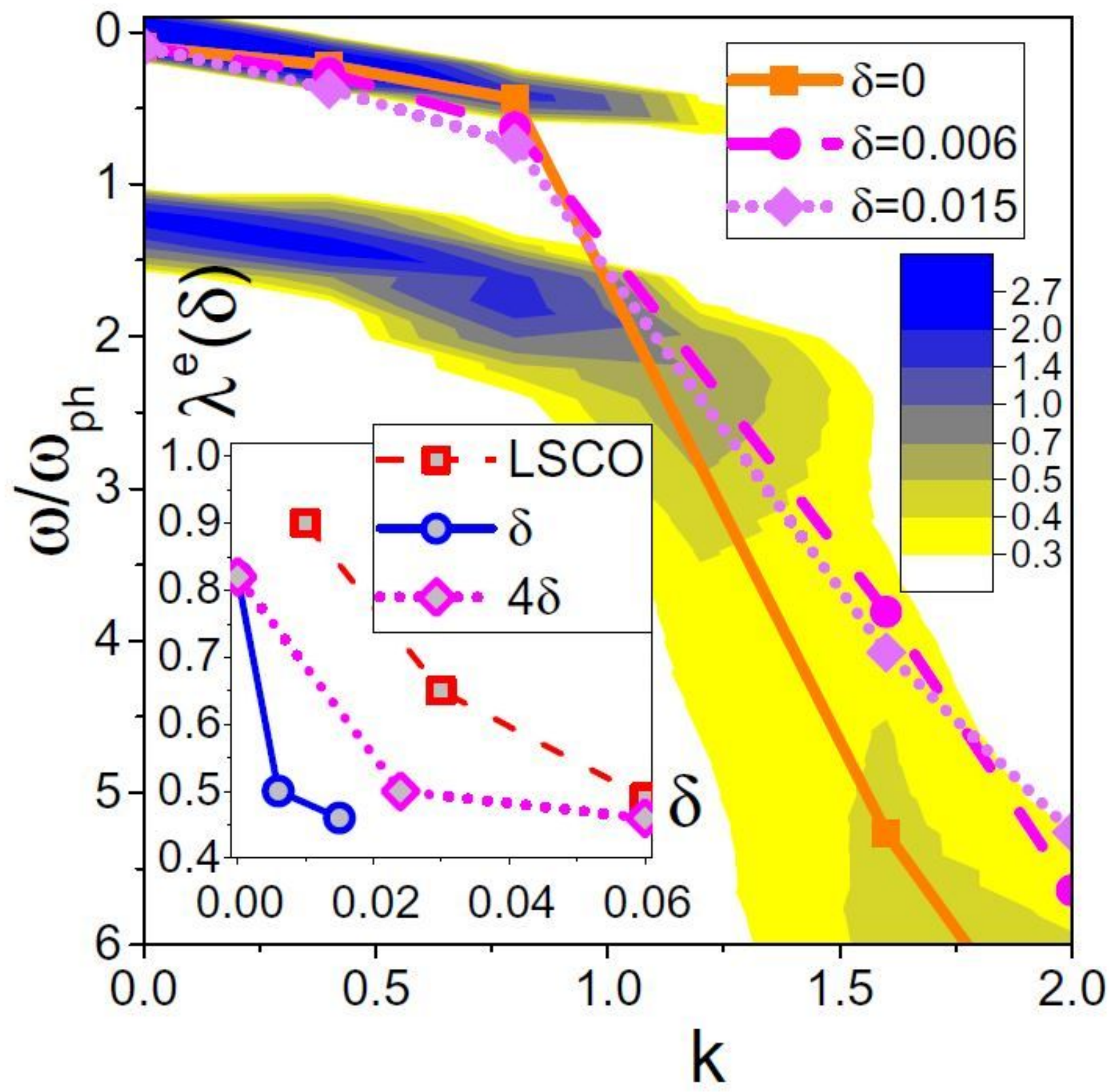

Figure 3

Contour plot of the spectral function intensity at $\nabla=3: 8 \otimes 10-4$ with blue/yellow color used for the large/small intensity. Symbols connected with lines mark locations of the spectral density maxima, see also Fig. 4 , for: $₫=3: 8 \otimes 10-4$ (squares connected by the solid line), $\rrbracket \approx 0: 006$ (circles connected by the dashed line), and $₫ \approx 0: 015$ (diamonds connected by the dotted line). In the inset we present the effective coupling constant 『e deduced from the scaling relation (2) using experimental data for LSCO [18] (squares connected by a dashed line) and locations of theoretical spectral density maxima in Fig. 4 (circles connected by a solid line). We also re-plot the same theoretical data by using $4 \llbracket$ for the horizontal 
axis (diamonds connected by a dotted line). Spectral densities were computed for self-energies evaluated

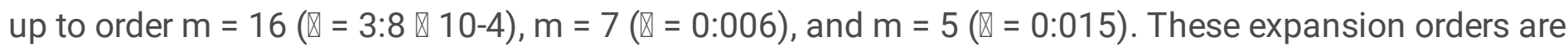
enough to have converged results for the corresponding carrier density (see Supplemental Material [33], Table I).

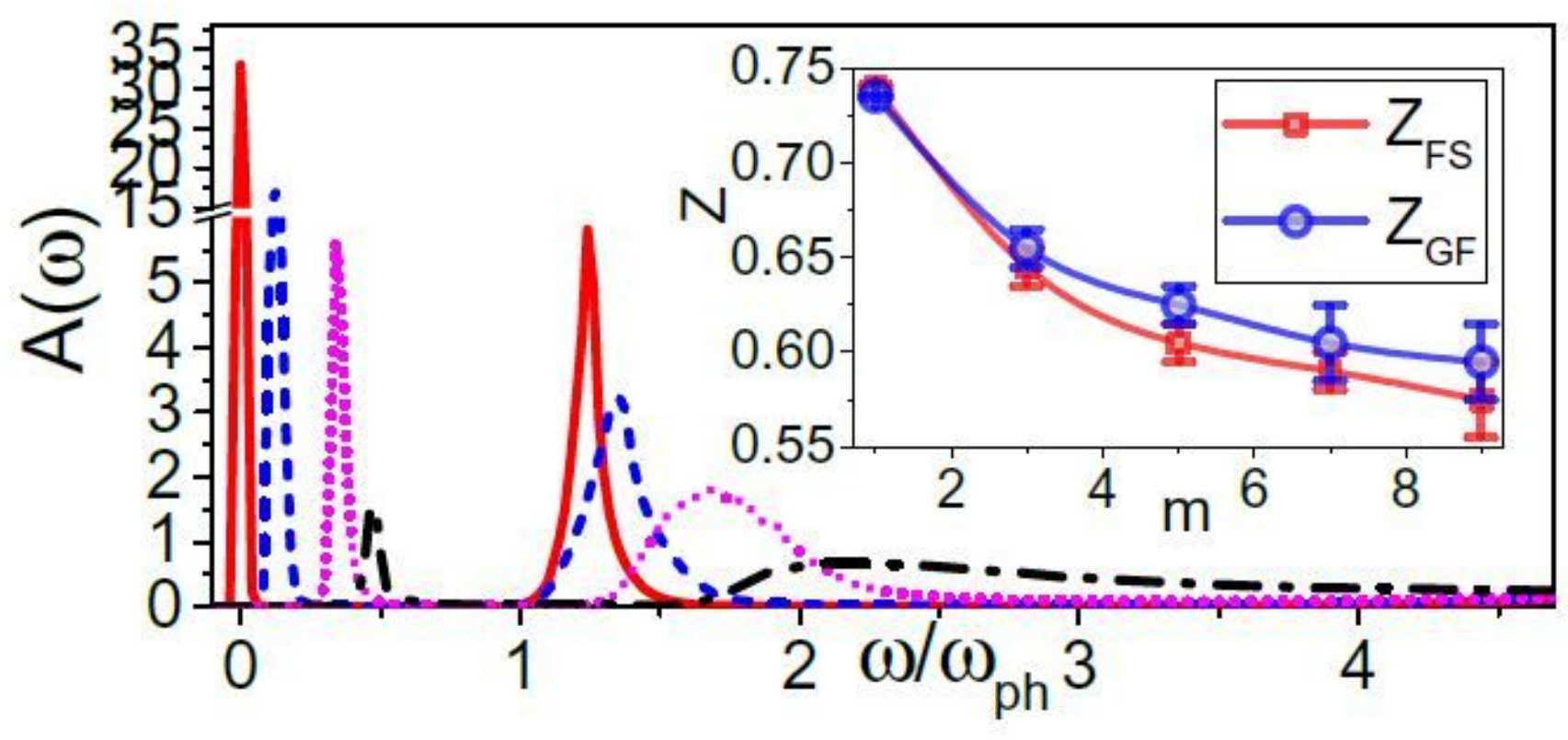

Figure 4

Spectral functions $A(\nabla)$ at different momenta for $\nabla=3: 8 \otimes 10-4$ from $m=16$ simulations: $q=(0 ; 0)$ (red solid line), $q=(\varangle / 8,0)$ (blue dashed line), $q=(2 \varangle / 8,0)$ (magenta dotted line), and $q=(3 \varangle / 8,0)$ (black dash-dotted line). Energy zero was set at the value of the QP dispersion relation at $q=0$. Inset: Order- byorder comparison between the two alternative procedures for computing the quasi-particle residue at $q=$ 0 : (i) using standard Fermi liquid relations at the Fermi surface, ZFS, and (ii) from the lowest-frequency peak in the spectral function, ZGF . 


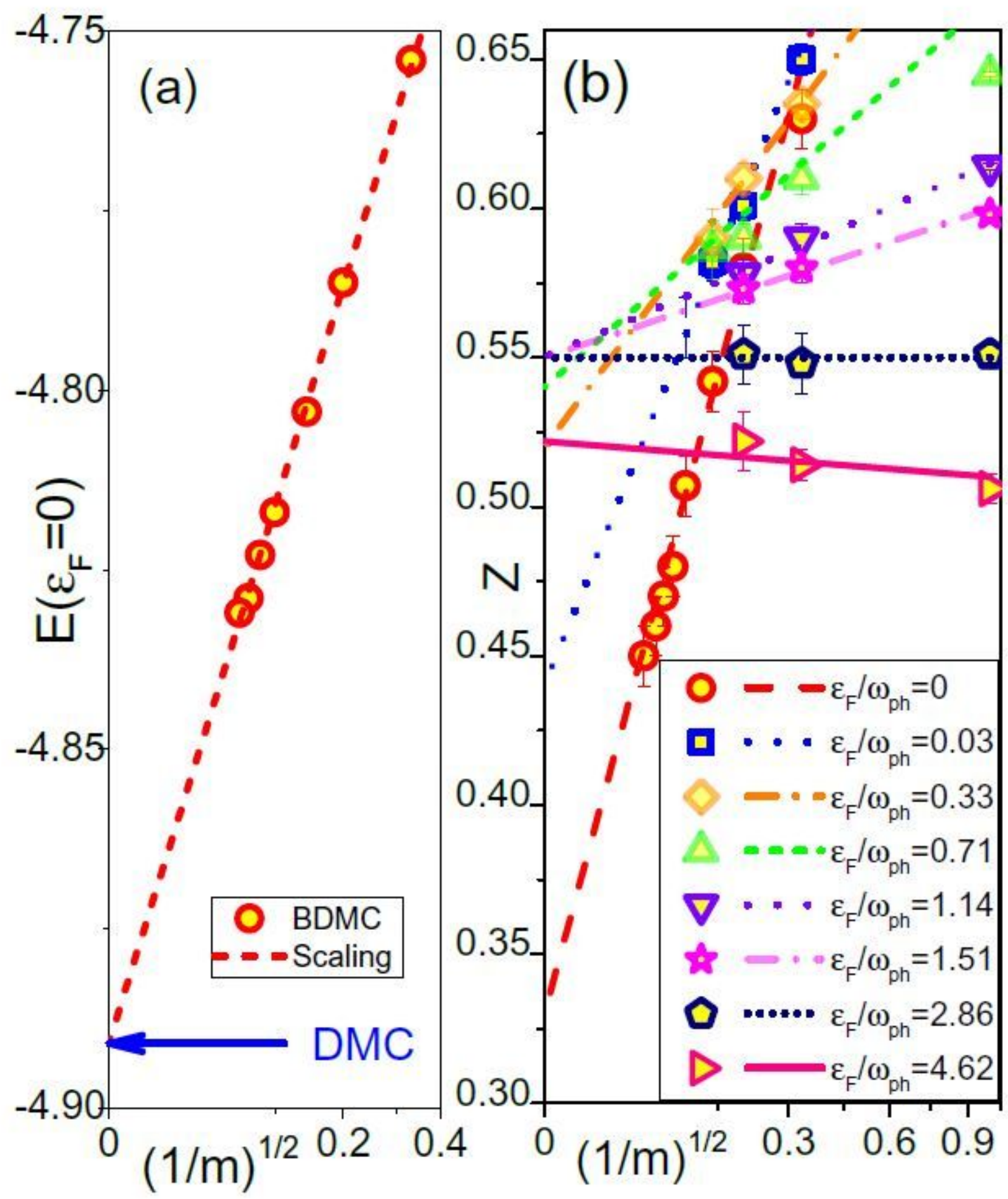

Figure 5

Finite expansion-order corrections to the polaron en- ergy (a) and QP residue (b) revealing linear scaling with $m-1 / 2$. (a) BDMC data (circles) and the scaling law $a+b / \sqrt{ } m$ (dashed line) for the ground state

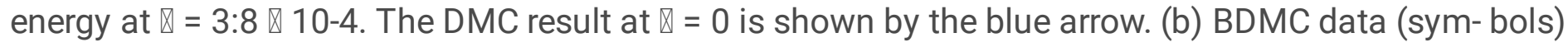
and the scaling laws $a+b / \sqrt{ } m$ (lines) for the quasi-particle residue. 


\section{Supplementary Files}

This is a list of supplementary files associated with this preprint. Click to download.

- supplement.pdf 